Erschienen in: Blühdorn, Hardarik/Breindl, Eva/Waßner, Ulrich W. (Hrsg.):

Text - Verstehen. Grammatik und darüber hinaus. - Berlin, New York: de

Gruyter, 2006. S. 205-221. (Institut für Deutsche Sprache. Jahrbuch 2005),

https://doi.org/10.1515/9783110199963.2.205

\author{
BARBARA HEMFORTH
}

\title{
Psycholinguistische Methoden zur Untersuchung des Satz- und Textverstehens
}

\begin{abstract}
Die zentrale Forschungsmethode in der Psycholinguistik ist das psychologische Experiment. Dadurch unterscheidet sich die psycholinguistische Forschung in mancher Hinsicht von anderen Gebieten der Sprachwissenschaften, in denen die Beobachtung natürlichen Vorkommens sprachlicher Phänomene eine deutlich größere Rolle spielt. Ich werde im folgenden Beitrag nach einer kurzen Definition psycholinguistischer Fragestellungen zunächst Experiment und Beobachtung einander gegenüberstellen und dabei dafür argumentieren, dass es sich hier um einander ergänzende Herangehensweisen handelt. Ich werde dann verschiedene psycholinguistische Experimentaltechniken vorstellen, von sehr einfachen Fragebogentechniken bis hin zu technisch höchst aufwendigen Methoden wie der Messung von Blickbewegungen oder von sprachspezifischen EEG-Mustern. Hier werde ich versuchen, deutlich zu machen, dass nicht die Kosten allein die Qualität einer Technik ausmachen, sondern die Angemessenheit für die Fragestellung.
\end{abstract}

\section{Psycholinguistische Fragestellungen}

Wenn ein zentraler Punkt bei der Auswahl einer experimentellen Technik die Angemessenheit für die Fragestellung ist, muss zunächst einmal geklärt werden, welche Art von Antworten in der psycholinguistischen Forschung gesucht wird. Hier ist eine wesentliche Voraussetzung, dass das Verstehen und die Produktion von sprachlichen Äußerungen als genuin kognitive Phänomene betrachtet werden. Damit zielt die psycholinguistische Forschung auf Verarbeitungsmodelle, die die kognitive Repräsentation sprachlichen Wissens sowie ein Prozessmodell der Verarbeitung dieses Wissens in den Mittelpunkt stellen. Dabei basieren sowohl Repräsentations- als auch Verarbeitungsmodelle auf allgemeinen Annahmen über die kognitive Architektur des Menschen. Dies schließt Annahmen über die Struktur der Wissensrepräsentation im Allgemeinen genauso ein wie solche über das Arbeitsgedächtnis, Aufmerksamkeit und vieles mehr. Aus diesen Grundannahmen ergibt sich zwangsläufig, dass eine psycholinguistische Theorie nach sprachübergreifenden Prinzipien sucht, eben solchen, die sich im Wesentlichen aus der kognitiven Architektur des Menschen ergeben. Sprachspezifische Unterschiede sollten dann allerdings in Interaktion mit der allgemeinen Architektur des Sprachverstehenssystems zu interessanten Unterschieden in der konkreten 
Verarbeitung von sprachlichen Äußerungen führen. Aus diesem Grund ist die deskriptive Erforschung verschiedenster Sprachen einer der Bereiche der allgemeinen Linguistik, die für die psycholinguistische Forschung von höchster Relevanz sind. Zu kurz kommt zur Zeit sicher häufig noch die Einbeziehung kulturspezifischer Einflüsse auf die Verarbeitung sprachlichen Wissens. Hier wird gerade in neuerer Zeit durch die verstärkte Ausrichtung der Forschung auf Dialoge und Texte (siehe etwa Pickering/Garrod 2004 oder Strohner im vorliegenden Band) erhöhter Bedarf an intensiverer Forschung über kulturspezifische Aspekte der Kommunikation erkennbar.

Ein sicherlich wichtiger Punkt ist, dass die aufzudeckenden Prinzipien universellen Charakter haben, dies unter der Annahme, dass die kognitive Architektur aller Menschen im Wesentlichen gleich ist. Das Ideal wäre ein Modell, in das sprach- und kulturspezifisches Wissen sowie interindividuelle Unterschiede etwa in der sprachlichen Kompetenz oder im Arbeitsgedächtnis als Parameter eingegeben werden, und das so konkretes sprachliches Verhalten vorhersagt. Von einem solchen Modell sind wir sicherlich noch weit entfernt. Die Annahme allerdings, dass sprachliches Verhalten durch möglichst allgemeine Prinzipien erklärt werden soll, die über Individuen und über individuelle sprachliche Äußerungen hinweg gültig sind, führt zu dem Primat des Experiments als Forschungsmethode in der psycholinguistischen Forschung.

\section{Experiment und Beobachtung}

Was versteht man unter einem Experiment? Üblicherweise ist dies das Herstellen einer Situation, in der eine oder mehrere Variablen oder Parameter variiert werden, während möglichst alle anderen kontrolliert werden. Dies hat im Idealfall den Vorteil, dass Veränderungen im Verhalten sich eindeutig auf die manipulierten Parameter zurückführen lassen. Durch die Einbeziehung vieler Probanden und vieler Instanzen einer Klasse sprachlicher Äußerungen wird versucht, individuelle Unterschiede (von Menschen genauso wie von Sätzen oder Texten) als „Zufallsrauschen“ herauszufiltern. Nur „überzufällige" Unterschiede, also solche, deren Zustandekommen durch zufälliges „Rauschen“ sehr unwahrscheinlich ist, werden als bedeutsam oder „signifikant" betrachtet. Der Nachteil allerdings ist, dass die experimentelle Situation gerade aufgrund dieser Beschränkungen oft sehr künstlich und wenig lebensnah ist.

Die Beobachtung richtet sich dagegen auf natürliches Verhalten in natürlichen Lebenssituationen bei möglichst geringer Beeinflussung des beobachteten Verhaltens. Dies hat den großen Vorteil, dass im Idealfall tatsächlich reales Verhalten beobachtet wird. Ein Nachteil ist allerdings, dass sich Ursache-Wirkungsverhältnisse selten klar herausfinden lassen, da im „wirklichen Leben" oft zu viele Parameter gleichzeitig variieren. Allerdings ist die Beobachtung real auftretender sprachlicher Phänomene natürlich die Basis 
jedes psychologischen Experiments, wenn auch eher als Popperscher Context of Discovery.

Eine auch für die psycholinguistische Forschung inzwischen viel genutzte Kombination aus „Beobachtung“ und statistischen Verfahren wird durch die Aufarbeitung großer Textkorpora möglich (siehe z. B. MacDonald/Pearlmutter/Seidenberg 1994). Korpusanalysen liefern wichtige Daten gerade für die Erklärung sprachspezifischer Unterschiede (siehe z. B. Desmet/de Baecke/Drieghe/Brysbeart/Vonk im Druck).

Andererseits sagen Korpusanalysen meist wenig über die kognitive Repräsentation der im Text dargestellten Situation aus. Sie sagen wenig darüber aus, welche Prozesse zu welchem Zeitpunkt beim Verstehen bzw. der Produktion des Textes zum Einsatz kommen. Sie enthalten meist überarbeitetes, editiertes Material, das frühe Verstehens- und Produktionsprozesse, die im eigentlichen Zentrum psycholinguistischer Forschung stehen, nicht widerspiegelt. Außerdem ist es bei komplexen Fragestellungen zuweilen nicht möglich, ausreichend viel Textmaterial zu finden (sparse data problem).

Als ein konkretes Beispiel für die Verwendung von Korpusanalysen in der psycholinguistischen Forschung möchte ich auf ein eigenes Forschungsprojekt zurückgreifen, das sich mit sprachspezifischen Unterschieden bei der Interpretation von Pronomen befasst (Hemforth/Colonna/Pynte/Scheepers/ Konieczny 2005). Dies ist ein für die Psycholinguistik besonders interessantes Phänomen, da gerade die Auflösung von Textverweisen (Referenzen) sehr häufig mehrdeutig ist. Betrachten wir etwa einen Satz wie (1). Hier ist das Pronomen er mehrdeutig insofern, als es der Briefträger oder der Postbote sein kann, der nach Hause ging:

\section{(1) Der Briefträger traf den Postboten, als er nach Hause ging.}

Wie wird dieses Pronomen interpretiert? Wir untersuchten Sätze in deutschen, französischen und englischen Korpora, in denen die Zuordnung der Pronomen eindeutig war. Dabei wählten wir jeweils zufällig fünfzig Sätze mit einem Subjekt und einem möglichen weiteren Antezedens im Matrixsatz. Es fanden sich folgende Verteilungen:

- im Deutschen nahmen 77\% der Pronomen das Subjekt als Antezedens (50 Sätze aus dem Frankfurter Rundschau Korpus);

- im Englischen nahmen 63\% der Pronomen das Subjekt als Antezedens (50 Sätze aus dem Wall Street Journal);

- im Französischen nahmen 100\% der Pronomen das Objekt als Antezedens (50 Sätze aus dem Le Monde Korpus).

Die eigentlich interessante Frage ist hier natürlich, inwiefern sich diese Verteilung im tatsächlichen Sprachverstehen widerspiegelt. Bevor wir dieser Frage mit typischeren psycholinguistischen Methoden nachgehen, möchte ich jedoch in einem kleinen Exkurs die spezifisch psycholinguistische Herangehensweise noch etwas genauer erläutern. 


\section{Exkurs: Beispiel für eine psycholinguistische Heran- gehensweise an eine linguistische Hypothese}

In diesem Beispiel möchte ich mich an einen Beitrag des vorliegenden Bandes anlehnen, den von Maria Thurmair, der in meines Erachtens klassisch linguistischer Weise eine sehr interessante These behandelt. Eine - unzulässig vereinfachte - Zusammenfassung wäre:

Hypothese:

Konjunktive dienen der Textstrukturierung, sie markieren eingebettete Texteinheiten.

In Thurmairs Beitrag wird anhand von Textstellen überzeugend dargelegt und belegt, in welcher Weise Konjunktive als linguistisches Mittel verwendet werden können, um eingebettete Texteinheiten zu markieren. Aus psycholinguistischer Perspektive stellt sich nun etwa die Frage, welche Auswirkungen dies auf den direkten Verstehensprozess beim Menschen haben kann. Wird diese Strukturierung direkt bei der Verarbeitung eines Textes wahrgenommen? Führt sie zu einer unterschiedlichen Repräsentation der im Text dargestellten Situation, einem anderen „Mentalen Modell“ (s. Schnotz im vorliegenden Band)? Präziser gesagt: Es stellt sich die Frage nach der Auswirkung der Verwendung von Konjunktiven einerseits auf den Verarbeitungsprozess und andererseits auf das Resultat des Textverstehens. So könnte man etwa annehmen, dass der Wechsel von Texteinheiten mit erhöhtem Verarbeitungsaufwand verbunden ist. Dies beträfe den Verarbeitungsprozess. Auf der Seite des Resultats der Verarbeitung müsste man wiederum vermuten, dass Entitäten, die derselben Texteinheit angehören, enger zusammenhängen als solche, die aus verschiedenen Texteinheiten stammen (siehe etwa die Folgen von Szenarienwechseln bei Sanford/Garrod 1981).

Ein psycholinguistisches Experiment zur Prüfung möglicher Unterschiede in der Repräsentation ließe sich etwa folgendermaßen konzipieren: Ich mache mir hier ein bekanntes sprachpsychologisches Phänomen zunutze, das als Priming oder Bahnung bezeichnet wird (für einen Überblick siehe Zwiserlood/Boelte 2002). Beim Priming führt die Verarbeitung eines Wortes zur Voraktivierung anderer Wörter, die mit diesem in engerem Zusammenhang stehen. Letztere können dann gegebenenfalls schneller verarbeitet werden.

Für ein psycholinguistisches Experiment müsste nun eine Reihe von Texten (hier mindestens zehn verschiedene) in jeweils zwei Ausprägungen konstruiert werden. In (2) findet sich ein Beispieltext:

(2a) 1. Sie stand am Herd und schälte die Kartoffeln.

2. Da kam er zur Tïr herein.

3. Er hätte schreckliche Kopfschmerzen.

4. Die Arbeit wïrde ihm schon lange zuviel.

5. Er ließ sich auf den Stuhl fallen. 


\section{(2b) 1. Sie stand am Herd und schälte die Kartoffeln.}

2. Da kam er zur Tür herein.

3. Er hatte schreckliche Kopfschmerzen.

4. Die Arbeit wurde ihm schon lange zuviel.

5. Er ließ sich auf den Stuhl fallen.

Jeweils eine Variante jedes Textes (fünf in jeder Ausprägung) würde dann einer Reihe von Probanden (hier wohl mindestens 40 Probanden) präsentiert. Eine ganze Reihe von weiteren Texten wäre zur Kontrolle verschiedenster Störfaktoren nötig. Nach jedem Text würde den Probanden nacheinander eine Liste von Wörtern wie (3) präsentiert. Ihre Aufgabe wäre es, anzugeben, ob das jeweilige Wort im Text enthalten war:

(3) Äpel

Kartoffeln

Kopfschmerzen

Sessel

Eine mögliche Vorhersage wäre: Wenn das Wort Kopfschmerzen direkt nach dem Wort Kartoffeln beurteilt werden soll, sollte das nach der Lektüre von Text (2b) schneller gehen als nach der von Text (2a), da nur in (2b) die Texteinheit fortgeführt wird, während im Text (2a) durch die Einführung des Konjunktivs in Satz (3) ein Wechsel der Texteinheit suggeriert wird. Der PrimingEffekt sollte bei Wörtern innerhalb einer Texteinheit größer sein als zwischen Wörtern verschiedener Texteinheiten.

Will man den Verarbeitungsprozess selbst untersuchen, könnte man etwa die Texte satzweise lesen lassen und dabei die Lesezeiten festhalten. Eine mögliche Vorhersage wäre hier, dass der Wechsel der Texteinheit in Satz 5 im Text (2a) zu erhöhten Lesezeiten führen sollte.

Dies waren zwei mögliche Beispiele für die Konzeption psycholinguistischer Experimente. Im Folgenden werde ich eine allgemeinere Klassifikation experimenteller Techniken in der psycholinguistischen Forschung diskutieren.

\section{Offline- und Online-Experimente}

Experimentelle Techniken unterscheiden sich darin, zu welchem Zeitpunkt sie Verarbeitungsprozesse bzw. deren Resultate überprüfen. Bei so genannten Offline-Verfahren wird das Textverständnis im Anschluss an den Verarbeitungsprozess überprüft. Online-Verfahren zeichnen sich dagegen dadurch aus, dass das Textverständnis während des Verarbeitungsprozesses selbst überprüft wird. (Man könnte hier die Skizze des Priming-Experimentes weiter oben als Offline-Experiment, das Lesezeit-Experiment dagegen als OnlineExperiment bezeichnen.) Tatsächlich handelt es sich hier aber um zwei Pole einer kontinuierlichen Skala, die auch von der Korngröße der Fragestellung abhängt. So spiegeln Satzlesezeiten oft nur unzureichend Verarbeitungspro- 
zesse wider, die sich etwa auf der Wort- oder Phrasenebene abspielen. Oft werden Reaktionszeitdaten als Online-Daten betrachtet, Interpretationen, Beurteilungen oder Fehler dagegen als Offline-Daten. Damit würde das Priming-Experiment weiter oben wiederum als Online-Verfahren klassifiziert, obwohl der Verarbeitungsprozess selbst hier nicht untersucht wird. Da Online-Verfahren oft technisch aufwändiger sind, werden sie häufig als qualitativ besser beurteilt, was zu einer immer wiederkehrenden Debatte darüber führt, welche Verfahren wirklich online sind. Diese Debatte ist allerdings nicht immer nützlich. Wenn, wie im obigen Beispielfall, tatsächlich das Resultat des Verarbeitungsprozesses die Forschungsfrage bestimmt, ergibt sich daraus zwangsläufig die Nützlichkeit eines Verfahrens, das eventuell technisch weniger aufwändig ist (was in diesem Fall allerdings nicht zutrifft).

\subsection{Fragebogentechniken}

Ich werde im Folgenden zwei Beispiele für Offline-Fragebogen-Untersuchungen aus einem eigenen Projekt präsentieren (Hemforth et al. 2005). Die Fragestellung betrifft hier, wie bei der Korpusanalyse weiter oben, die Interpretation von Pronomen in Sätzen wie $(4 a / b)$. Probanden erhielten einen Fragebogen mit jeweils 8 Sätzen in einer der beiden Varianten, gemischt mit ca. 60 Füllsätzen anderer Struktur. Aufgabe der Probanden war es, die Lücke in der Teilparaphrase jeweils unter dem Experimentalsatz zu füllen. Ziel dieses Experimentes war es, einen ersten Eindruck davon zu bekommen, welches der beiden möglichen Antezedenzien hier bevorzugt wird. Um auszuschlieBen, dass Plausibilitätsunterschiede in der Zuordnung des Temporalsatzes zum Subjekt oder Objekt des Satzes eine Rolle spielen, wurden die beiden Rollen bei der Hälfte der Probanden vertauscht (hier hieß es dann: Der Straßenfeger traf den Briefträger, bevor er nach Hause ging. Und: Der Straßenfeger traf den Briefträger. Dann ging er nach Hause.):

(4a) Der Briefträger traf den Straßenfeger, bevor er nach Hause ging. Der__ ging nach Hause.

(4b) Der Briefträger traf den Straßenfeger. Dann ging er nach Hause. Der__ ging nach Hause.

Das Ergebnis dieses Experiments findet sich in der Abbildung 1a.

Deutsche Probanden zeigten sowohl für die Verweise innerhalb von Sätzen (4a) als auch für die Verweise zwischen Sätzen (4b) eine klare Präferenz für das Subjekt als Antezedens. Dies ist nun insofern besonders interessant, als wir ein maximal vergleichbares Experiment mit den Sätzen (5a/b) für das Französische durchgeführt haben:

(5a) Le facteur a rencontré le balayeur avant qu'il rentre à la maison. Le est rentré à la maison.

(5b) Le facteur a rencontré le balayeur. Puis il est rentré à la maison. Le est rentré à la maison. 
Abb. 1a

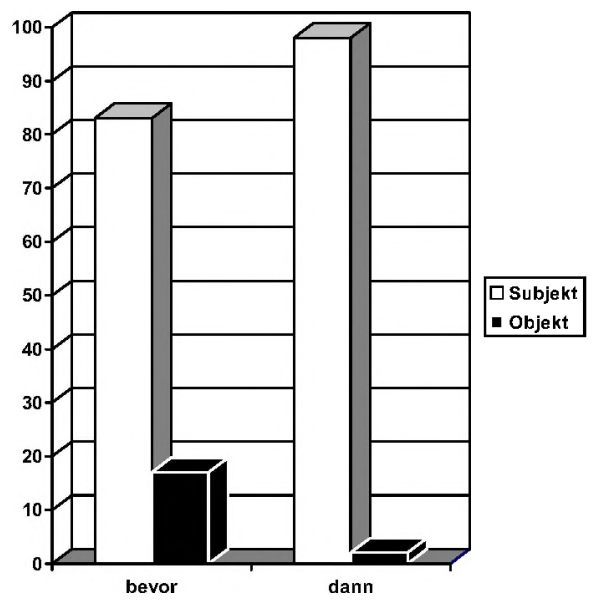

Hier fand sich bei Verweisen zwischen Sätzen wie in (5b) eine der im Deutschen gefundenen vergleichbare Präferenz für das Subjekt. Bei Verweisen innerhalb von Sätzen wie in (5a) jedoch wurde eindeutig das Objekt als Antezedens präferiert (Abb. 1b).

Abb. 1b

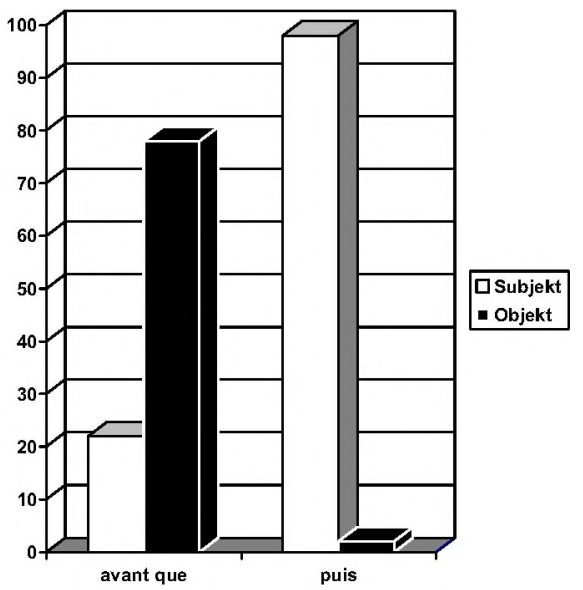

Diese Präferenz entspricht deutlich den Ergebnissen der Korpusanalysen. Eine mögliche Erklärung für diese sprachspezifischen Unterschiede ist die Tatsache, dass im Französischen eine starke, nahezu grammatikalisierte Alternative für die Subjektbindung besteht. Hier würde eher eine Infinitivkonstruktion wie in (6) verwendet, die nur das Subjekt als Antezedens der Null-Anapher zulässt:

(6) Le facteur a rencontré le balayeur avant de rentrer à la maison.

Das hier gezeigte Verfahren ist sehr nützlich, wenn grobe Unterschiede in Interpretationspräferenzen aufgedeckt werden sollen. Feinere Graduierungen 
sind jedoch hiermit nur schwer zu entdecken. Hier wurde in den letzten Jahren ein Verfahren aus der Psychophysik der psycholinguistischen Forschung angepasst, das heute vielfach eingesetzt wird. Die so genannten Magnitude Estimations wurden ursprünglich von Stevens (1957) zur Untersuchung des Verhältnisses von physikalischer Stimulusintensität und der erlebten Intensität entwickelt. Stevens ließ Probanden die Intensität eines Stimulus im Vergleich zu einem Referenzstimulus einschätzen (etwa: Ton A ist halb so laut, doppelt so laut etc. wie Ton B). Die subjektive Wahrnehmung von Helligkeit oder Lautstärke steht hier in einem exponentiellen Verhältnis zur physikalischen Intensität, das so genannte psychophysische Gesetz.

Ein ähnliches Verfahren wird für linguistische Urteile verwendet (Bard et al. 1996; Cowart 1997). Probanden weisen hier einem Referenzsatz oder Text eine beliebige Zahl zu und beurteilen Experimentalsätze oder -texte im Vergleich zu diesem Satz. Die Daten werden dann standardisiert und logarithmiert, um eine lineare Beziehung zu erhalten.

Wir verwendeten dieses Verfahren, um zu überprüfen, ob im Deutschen tatsächlich in Sätzen wie (7a/b) das Subjekt präferiertes Antezedens für ein Pronomen ist, oder vielleicht einfach die Nominalphrase, die an der ersten Position im Satz steht (7c/d) (Konieczny/Hemforth 2001):

(7a) Die Schauspielerin faszinierte den Regisseur, als er sich vorstellte.

(7b) Die Schauspielerin faszinierte den Regisseur, als sie sich vorstellte.

(7c) Den Regisseur faszinierte die Schauspielerin, als sie sich vorstellte.

(7d) Den Regisseur faszinierte die Schauspielerin, als er sich vorstellte.

Abbildung 2 zeigt die standardisierten und logarithmierten Urteile der Probanden. Es zeigt sich, dass, abgesehen von einer Präferenz für die kanonische Subjekt-Objekt-Abfolge, Sätze immer dann mit höheren Akzeptabilitätswerten versehen wurden, wenn das Antezedens für das Pronomen am Satzanfang (in einer möglichen Topik-Position) stand:

Es kommen natürlich noch weitere Fragebogentechniken zu Einsatz, wie Multiple Choice Techniken (8), eine Rangordnung von Urteilen (9) und vieles mehr. Auf all diese Möglichkeiten einzugehen erlaubt hier jedoch der zur Verfügung stehende Raum nicht:

(8) Der Briefträger traf den Straßenfeger, bevor er nach Hause ging.

Wer ging nach Hause? Der Briefträger Der Straßenfeger

(9) Die Briefträgerin traf den Straßenfeger, bevor er nach Hause ging.

$\begin{array}{lr}0-1 & \text { völlig } \\ \text { völlig } & \text { inakzeptabel }\end{array}$

Will man nun wissen, an welcher Stelle im Text oder im Satz bestimmte Faktoren die Verarbeitung beeinflussen, so muss man auf andere Techniken zurückgreifen, da die bisher dargestellten Verfahren nur ein Maß für die Gesamtinterpretation liefern. Diesen so genannten Online-Verfahren werden wir uns im nächsten Abschnitt zuwenden. 

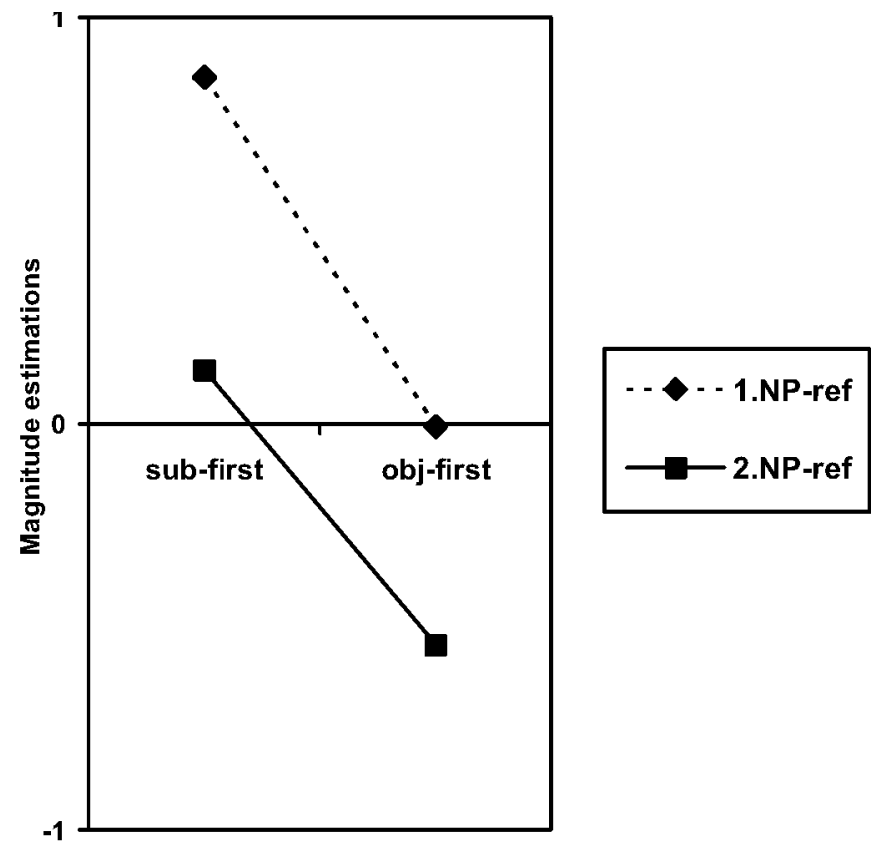

Abb. 2

\subsection{Online-Techniken: Selbstbestimmtes Lesen und Blickbewegungsmessungen}

Die meisten Online-Verfahren zum Textverstehen sind als Leseexperimente ausgerichtet. Dabei wird die Zeit, die für die Verarbeitung einer bestimmten Region verwendet wird, als Maß für den mit der Verarbeitung verbundenen kognitiven Aufwand betrachtet. Just/Carpenter/Wooley (1982) untersuchten verschiedene Präsentationstechniken und stellten dabei fest, dass die valideste Methode die so genannte Moving-Window-Technik sei. Hier wird das Sprachmaterial zunächst durch Bindestriche oder eine andere Maskierung ersetzt (jeder Buchstabe entspricht einem Bindestrich). Durch das Betätigen einer Taste wird dann zunächst der Teil der Maskierung, der der ersten darzubietenden Einheit entspricht, durch tatsächlichen Text ersetzt. Drückt der Proband wieder auf die Taste, verschwindet die Texteinheit und die nächste erscheint auf dem Bildschirm (10). Die Texte können wortweise dargeboten werden wie in (10) aber auch phrasenweise oder satzweise:

(10) Der Straßenfeger traf die Briefträgerin, als er/sie nach Hause ging.

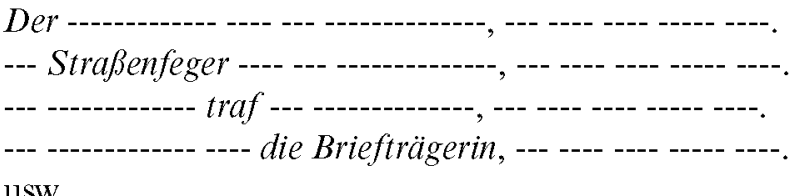

usw. 
Eine Standardkritik gegen dieses Verfahren ist, dass es sich hier sicherlich nicht um natürliches Lesen handelt, da nicht der ganze Text auf einmal zu sehen ist und da Probanden Tasten betätigen müssen, damit sie weiterlesen können. Wir werden auf diese Kritik zu einem späteren Zeitpunkt noch einmal zurückkommen. Zunächst möchte ich jedoch ein Verfahren vorstellen, das als ökologisch validere Alternative eingeführt wurde: die Analyse von Blickbewegungsdaten. Im Gegensatz zu Leseexperimenten, die mit frei verfügbaren Softwarepaketen auf nahezu jedem Laptop durchzuführen sind (das Paket DMDX von Ken und Jonathan Forster kann man sich auf der Webseite $\mathrm{http} /$ www.u.arizona.edu/ kforster/dmdx/dmdx.htm frei herunterladen), verlangen Blickbewegungsexperimente einiges Knowhow und vor allem teils recht kostenaufwändige Laborausstattung.

Tatsächlich ist es aber so, dass die Messung von Blickbewegungen es erlaubt, den Leseprozess relativ ungestört von zusätzlichen Aktionen (wie Tastendrücken) zu untersuchen. Zudem lassen sich hier verschiedene Maße zusammenfassen, die Einblick in unterschiedliche Zeitpunkte des Analyseprozesses erlauben. Rayner (1992) gibt hier einen ausgezeichneten Überblick. Abbildung 3 zeigt eine mögliche Abfolge von Blickbewegungen beim Lesen eines Satzes. Die kritische, das heißt hier interessante Region ist mit einem Kasten unterlegt:

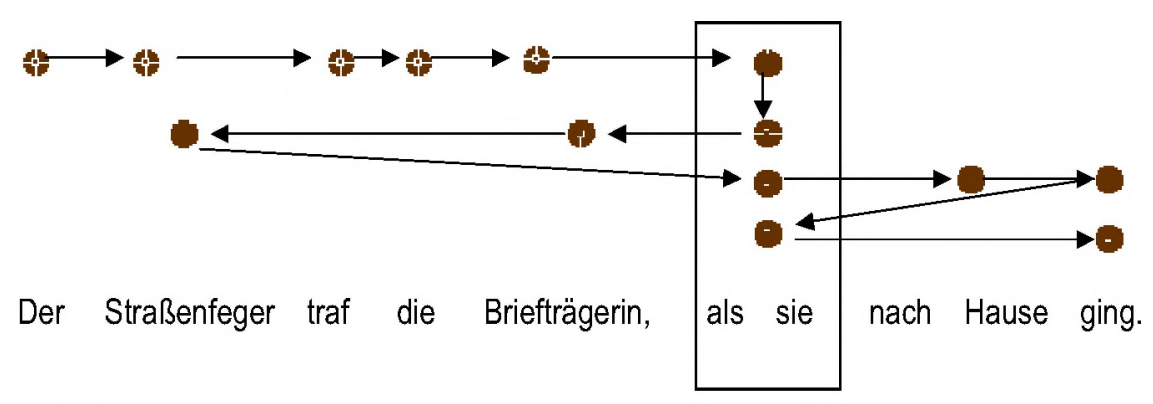

Abb. 3

Besonders frühe Prozesse (meist lexikalischer Art) finden sich oft in der ersten Fixation der kritischen Region. Ein typisches Maß für kognitiven Aufwand sind so genannte Gaze Durations, die Zeit zwischen dem ersten Eintreten und Verlassen der kritischen Region (das entspräche hier den ersten beiden Fixationen auf der kritischen Region). Manchmal allerdings werden gerade bei besonders schwierigen Sätzen die kritischen Regionen nur sehr kurz fixiert. Meist folgt dann ein Rücksprung in eine frühere Region, mit dem die Verstehensschwierigkeit gelöst werden soll. Ein Maß für solche Fälle sind Regression Path Durations (Konieczny/Hemforth/Scheepers/Strube 1997), die alle Fixationen vom ersten Eint reten in die kritische Region bis zum ersten Blicksprung nach rechts aus der Region einschließen, auch solche auf frühere 
Regionen im Text. Recht späte Prozesse werden durch Total Reading Times erfasst, die die gesamte auf der kritischen Region verbrachte Zeit einschließen (hier alle vom Kasten umschlossenen Fixationen). Abbildung 4 zeigt Total Reading Times auf der kritischen Pronomenregion für Sätze, wie sie für das Magnitude-Estimations-Beispiel verwendet wurden. Hier zeigt sich wie oben ein Topikeffekt: Die Lesezeiten sind kürzer, wenn sich das Pronomen auf die erste NP im Satz bezieht, unabhängig davon, ob es sich um das Subjekt oder das Objekt handelt (Hemforth/Konieczny/Leiss in Vorb.).

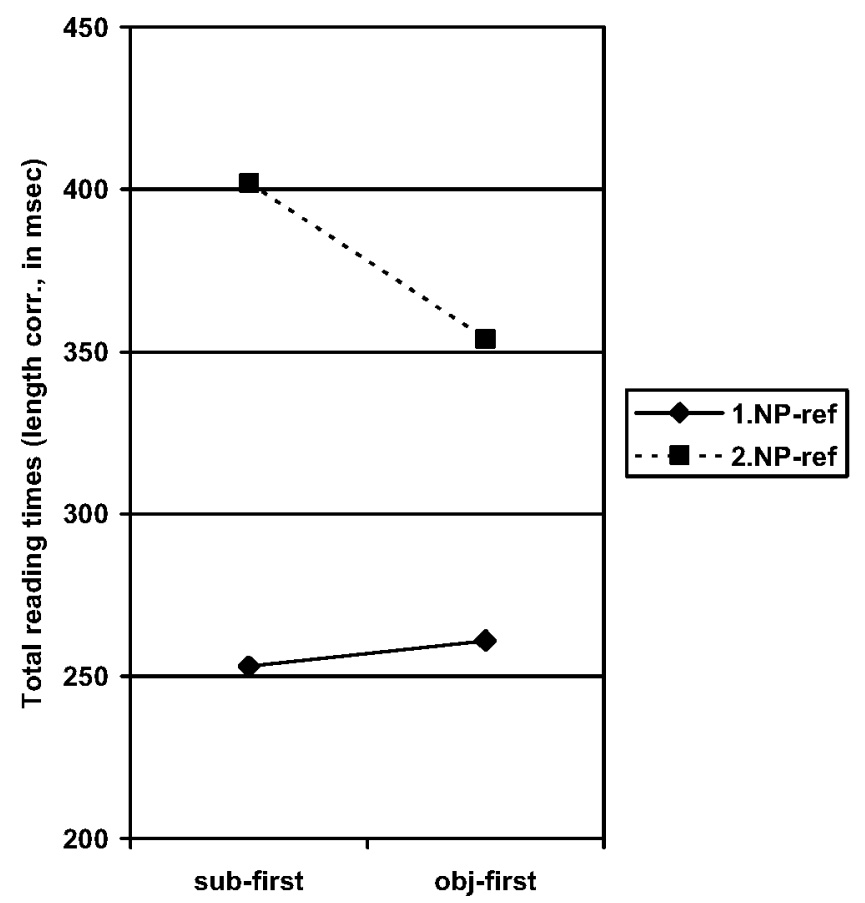

Abb. 4

Mitchell (2004) führt in einem ausgezeichneten historischen Überblick aus, dass oft wichtige Befunde zunächst mit einfacheren Lesezeitexperimenten entdeckt und dann mit Blickbewegungsexperimenten (und in neuerer Zeit mit ERP-Experimenten, siehe unten) validiert wurden. Er bezweifelt, dass die technisch aufwändigeren Methoden tatsächlich auch den erwünschten Erkenntnisfortschritt bringen, insofern als hier neue Phänomene entdeckt würden, die mit einfacheren Methoden nicht zu finden wären. Zudem stellt er zu Recht fest, dass die Notwendigkeit, den Kopf der Probanden bei Blickbewegungsexperimenten mechanisch zu fixieren, eine natürliche Lesesituation nur sehr eingeschränkt zulässt.

Bisher habe ich nur Methoden zum Leseverständnis vorgestellt. In neuerer Zeit wird jedoch mit dem Visual World Paradigm ein Verfahren eingesetzt, 
das es erlaubt, Interpretationen beim Verstehen gesprochener Sprache online zu untersuchen (Tanenhaus/Spivey-Knowlton/Eberhard/Sedivy 1995). Hier werden ebenfalls Blickbewegungen festgehalten, jedoch nicht beim Lesen, sondern beim Betrachten von Bildern oder realen Szenen. Es zeigt sich dabei, dass die Blickbewegungen auditiv dargebotenen sprachlichen Äußerungen folgen. So kann man etwa feststellen, dass Probanden, wenn sie beim Betrachten eines Bildes wie in Abbildung 5 die NP der Briefträger hören, zunächst verstärkt auf den Briefträger schauen, wenn sie dann trifft den Straßenfeger hören, schauen sie verstärkt auf den Straßenfeger. Interessanterweise entsprechen die Blickbewegungsmuster beim Hören des Pronomens dann den sprachspezifischen Präferenzen: Im Deutschen schauen die Probanden eher auf das Subjekt des Satzes, hier den Briefträger, im Französischen eher auf das Objekt, hier den Straßenfeger (Hemforth/Colonna/Scheepers/Pynte/ Konieczny 2005).

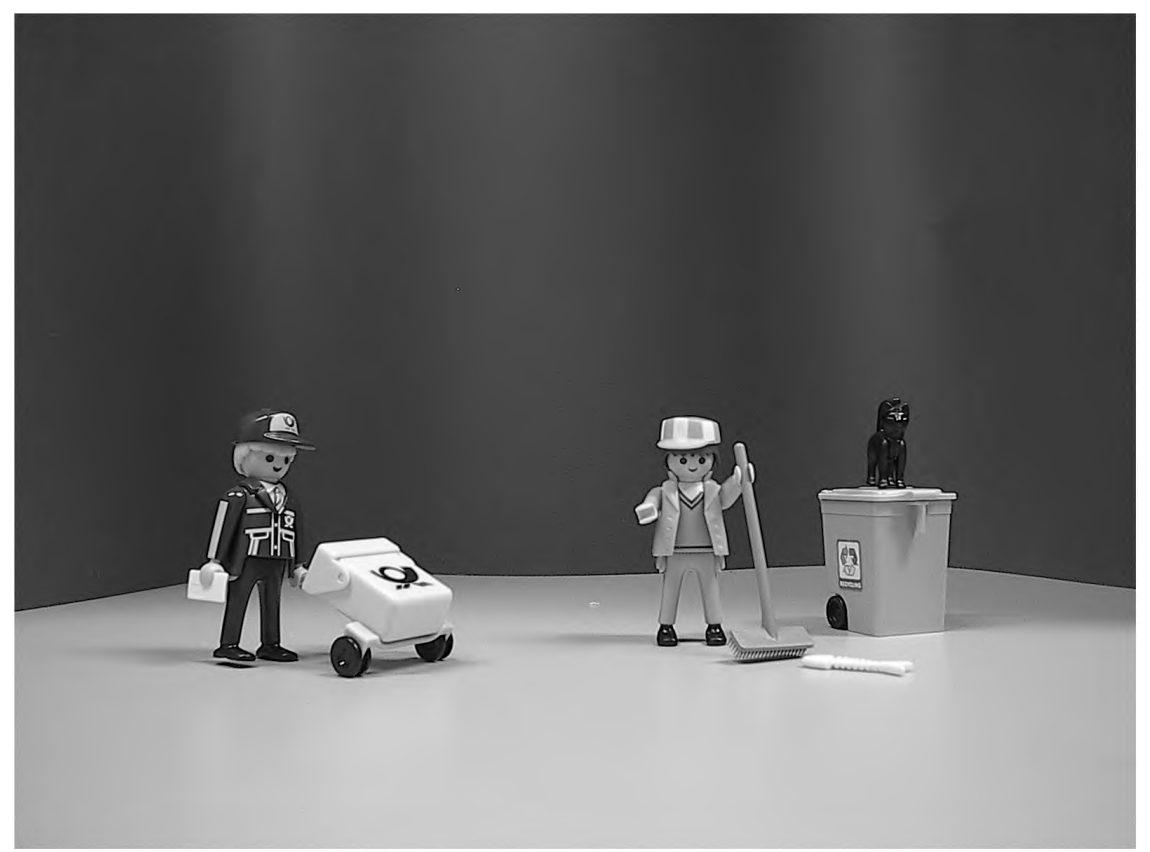

Abb. 5

Das Verstehen gesprochener Sprache ist ein klassisches Stiefkind psycholinguistischer Forschung, da hier bisher geeignete Online-Methoden fehlten, die nicht zu sehr in den Verstehensprozess eingreifen. Abgesehen von Blickbewegungsexperimenten im Visual World Paradigm sind hier vor allem neuropsychologische Techniken viel versprechend, die im folgenden Abschnitt behandelt werden. 


\section{Neuropsychologische Techniken}

Die hier sicherlich zur Zeit interessanteste Methode sind ereigniskorrelierte Potentiale (EKPs; im Englischen Event Related Potentials, ERPs). Hier werden EEG-Muster zeitlich abgestimmt auf ein bestimmtes sprachliches Ereignis festgehalten. Je nach Ausstattung des Labors werden 16, 32, 64 oder 128 Elektroden am Schädel des Probanden befestigt (meist geschieht dies mit einer Art Badekappe, in die die Elektroden eingebaut sind). In den Experimenten wird typischerweise eine Experimentalbedingung mit einer Kontrollbedingung verglichen. Die Experimentalbedingungen weisen oft spezifische Potentialverschiebungen auf, die in einem bestimmten Zeitfenster zu finden sind sowie eine bestimmte topographische Verteilung und eine bestimmte Polarisierung aufweisen.

Beispiele hierfür sind die Early Left Anterior Negativity (ELAN), ein negativer Ausschlag nach etwa 200 Millisekunden, der sich vor allem an Elektroden im vorderen linken Schädelbereich zeigt (der Sammelband von Friederici 1999 gibt hier einige Beispiele, besonders ihr eigener Beitrag), die N400Komponente, eher an zentralen Elektroden, und die P600-Komponente, eher im posterioren Schädelbereich. Oft werden verschiedene Komponenten mit unterschiedlichen sprachlichen Teilprozessen in Verbindung gebracht. Die ELAN-Komponente mit syntaktischer Analyse und Aufbau von Phrasenstrukturen, die N400-Komponente mit semantisch/lexikalischen Prozessen und die P600-Komponente mit syntaktischen Prozessen (vor allem Kongruenzprüfung) und Reanalyse. Einen guten Überblick bietet hier auch Berkum (2004).

Ein Beispiel für spezifische Komponenten bei der Verletzung von referentiellen Constraints findet sich bei Hemforth/Frenck-Mestre (2005). Wir verglichen ERP-Muster beim Lesen von französischen Sätzen wie (11a/b):

(11) Les bergères couraient vite quand (a) elles / (b) ils rattrapaient le troupeau. [Die Schäferinnen rannten schnell, als sie (fem/masc) die Herde wieder einfingen.]

Les bergères couraient vite. Enfin (a) elles / (b) ils rattrapaient le troupeau. [Die Schäferinnen rannten schnell. Endlich fingen sie (fem/masc) die Herde wieder ein.]

Fand sich hier das Antezedens nicht im Matrixsatz (die jeweiligen b-Versionen), so zeigte sich ein typisches P600-Muster (siehe Abbildung 6; Positivierungen werden hier konventionsgemä $\beta$ nach unten abgetragen). Hieraus könnte man schließen, dass entweder die Verletzung referentieller Bezüge hier ein eher syntaktisches Phänomen ist (siehe Osterhout/Mobley 1995) oder dass syntaktische und Diskursprozesse zu ähnlichen Mustern führen (siehe Berkum 2004):

Will man nicht nur verschiedene sprachspezifische Komponenten in EEGMustern extrahieren, sondern die Prozesse im Gehirn auch lokalisieren, so 


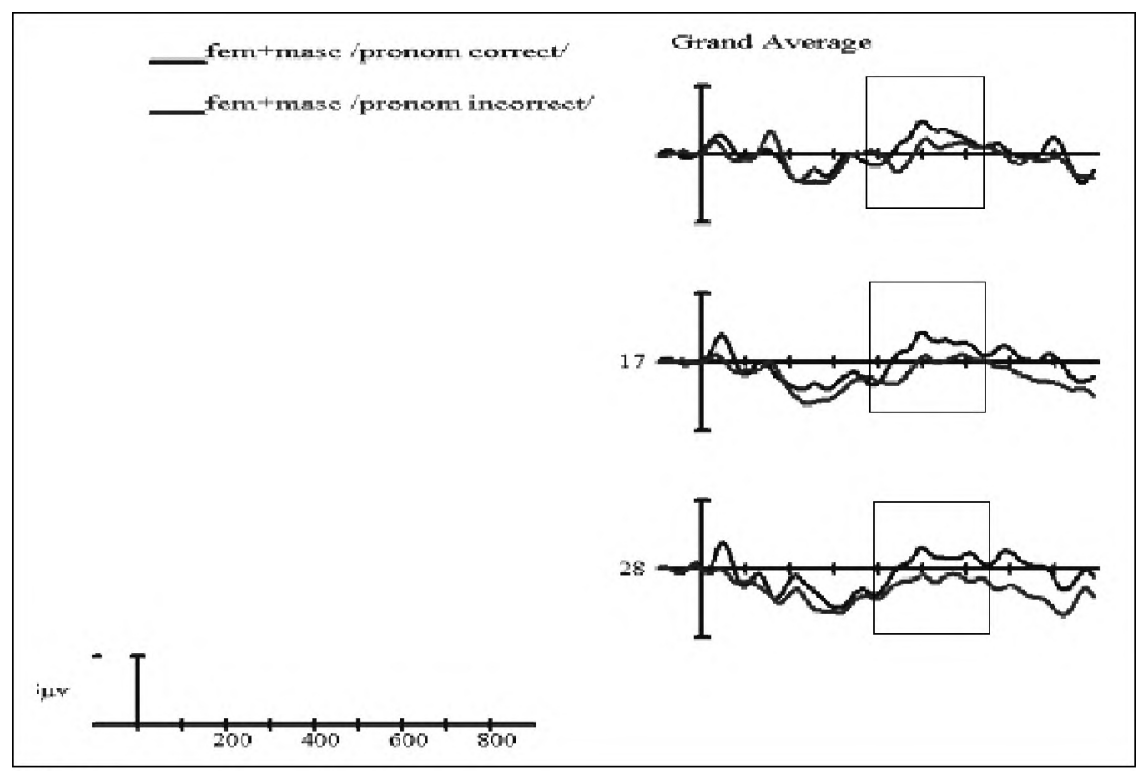

Abb. 6

muss man auf bildgebende Verfahren zurückgreifen, die meist mit der belegten Annahme arbeiten, dass erhöhte Aktivität in einem Hirnbereich mit verstärkter Durchblutung oder erhöhtem Sauerstoffverbrauch verbunden ist. Diese erhöhte Aktivität kann man etwa in der funktionellen Magnetresonanztomographie (fMRT) messen. Das übliche experimentelle Verfahren ist hier die Subtraktionsmethode, in der eine experimentelle Aufgabe, die lokalisiert werden soll, mit einer Kontrollaufgabe verglichen wird, die bis auf die interessierende Teilaufgabe möglichst ähnlich ist. Die Aktivierungen bei der Kont rollaufgabe werden dann von der Experimentalaufgabe subtrahiert. Was bleibt, stellt die spezifische Aktivierung für den interessierenden Prozess dar.

Ein Beispiel für ein solches Experiment findet sich bei Dogil et al. (2002). Hier wurden den Probanden einmal Wortlisten wie in (12) und einmal Sätze wie in (13) präsentiert. Die Aufgabe der Probanden war es, in Wortlisten wie in (12) das zweite Wort an den Anfang zu setzen, oder Sätze wie in (13) mit einer anderen Konstituente als der gegebenen zu beginnen:

(12) Hotelführer Museumsführer Kunstreiseführer Abfallgebühren Parkhausgebühren Leihgebühren Orangenbäume Haselnussbäume Apfelbäume Abenteuerroman Liebesroman Kriminalroman

(13) Juristen haben sich mit dieser Frage beschäftigt. Ein Aktivist hat sich an den Gitterzaun gekettet. Auf einem Dachboden haben sich Marder einquartiert. Uber dem Gebirge haben sich Wolken gebildet. 
Bei der spezifisch syntaktischen Aufgabe fanden sich Aktivierungen im Broca-Areal, im Wernicke-Areal, im dorso-lateralen präfrontalen Cortex (DPF), im anterioren cingulären Cortex (ACC), sowie überraschenderweise im Cerebellum (Kleinhirn).

Es sollte hier hinzugefügt werden, dass sowohl für EEG-Experimente als auch für fMRT-Experimente gilt, dass die Versuchsbedingungen sehr weit von natürlichen Sprachverarbeitungsumgebungen entfernt sind. Das starke Rauschen der Signale macht es nötig, dass über eine Vielzahl von Äußerungen gleicher Art gemittelt wird (in unserem Anaphernexperiment waren es 40 Sätze pro Bedingung). Die Probanden verbringen vergleichsweise viel Zeit im Labor (durchschnittlich 2 Stunden im Vergleich zu etwa einer halben Stunde in einem üblichen Lesezeitexperiment). Dabei wird die meiste Zeit auf technische Vorbereitungen verwendet.

\section{Schlussfolgerungen}

Wir haben gesehen, dass sich in der Psycholinguistik ein sehr weites Methodeninventar findet (das ich hier bei weitem nicht erschöpfend geschildert habe). Hier bieten sich je nach Fragestellung verschiedene experimentelle Techniken an. Jede dieser Techniken erlaubt Antworten auf unterschiedliche Fragen:

- Offline-Fragebögen: Welche Interpretationen werden bevorzugt, welche Formen des Ausdrucks sind mehr oder weniger akzeptabel?

- Reaktionszeitexperimente: Welche Formen des Ausdrucks sind kognitiv aufwändiger?

- ERPs: Welche Arten von Teilprozessen sind an der Verarbeitung des Textes beteiligt?

- fMRTs: Welche Hirnregionen sind beteiligt, und damit (falls bekannt), welche funktionalen Komponenten?

Das Vorhandensein von Blickbewegungslabors, ERP-Labors oder der Zugang zu fMRTs führt allerdings zuweilen dazu, dass eine technisch und finanziell zu aufwändige Methode angewendet oder zuweilen auch eingefordert wird, dass also mit Kanonen auf Spatzen geschossen wird. Dies heißt auf keinen Fall, dass diese Methoden nicht zu interessanten, neuen, und anders nicht zu untersuchenden Erkenntnissen führen. Es muss halt nur nicht immer und für jede Fragestellung die aufwändigste Methode zum Einsatz kommen.

Vergleichbar ist die Gefahr, dass das Vorhandensein entsprechender technischer Ausrüstung den Blick auf praktisch relevante Fragen verstellt, da lieber Fragen bearbeitet werden, die der entsprechenden Technik zugänglich sind: Wenn man einen Hammer hat, sieht jedes Problem wie ein Nagel aus. Oder besser: Wenn man einen Hammer hat, sucht man nur noch nach Problemen, die wie Nägel aussehen. 


\section{Literatur}

Bard, Ellen G./Robertson, Dan/Sorace, Antonella (1996): Magnitude estimation of linguistic acceptability. In: Language 72, S. 32-68.

Berkum, Jos van (2004): Sentence Comprehension in a Wider Discourse: Can we use ERPs to keep track of things? In: Carreiras, Manuel/Clifton Jr., Charles (2004) (ed.): The on-line study of sentence comprehension: Eyetracking, ERPs and beyond. New York: Psychology Press. S. 229-270.

Cowart, Wayne (1997): Experimental Syntax: Applying Objective Methods to Sentence Judgments. Thousand Oaks, CA: Sage Publications.

Desmet, Timothy/de Baecke, Constantijn/Drieghe, Denis/Brysbeart, Marc/Vonk, Wietske (im Druck): Relative clause attachment in Dutch: On-line comprehension corresponds to corpus frequencies when lexical variables are taken into account. In: Language and Cognitive Processes.

Dogil, Grzegorz/Ackermann, Hermann/Grodd, Wolfgang/Haider, Hubert/Kamp, Hans/ Mayer, Jörg/Riecker, Axel/Wildgruber, Dirk (2002): The Speaking Brain. In: Journal of Neurolinguistics 15 (1), S. 59-90.

Friederici, Angela D. (1999): The neurobiology of language comprehension. In Friederici, Angela D. (Hg.): Language comprehension: A biological perspective ( $2^{\text {nd }}$ ed.). Berlin: Springer. S. 101-132.

Hemforth, Barbara/Colonna, Saveria/Pynte, Joël/Scheepers, Christoph/Konieczny, Lars (2005): Pronoun resolution across languages: The role of distributional differences between French, German, and English. Poster presented at CUNY, 21. März - 2. April 2005, Tucson, Arizona.

Hemforth, Barbara/Frenck-Mestre, Cheryl (March, 2005): Anaphor resolution within and across sentences: An ERP-study. Paper presented at CUNY, 21. März-2. April 2005, Tucson, Arizona.

Hemforth, Barbara/Konieczny, Lars/Leiss, Myrto (in Vorb.): Topic effects in pronoun resolution.

Just, Marcel A./Carpenter, Patricia. A./Wooley, J. D. (1982): Paradigms and Processes in Reading Comprehension. In: Journal of Experimental Psychology: General 111/2, S. 228-238.

Konieczny, Lars/Hemforth, Barbara/Scheepers, Christoph/Strube, Gerhard (1997): The role of lexical heads in parsing: evidence from German. In: Language and Cognitive Processes 12 , S. 307-348.

Konieczny, Lars/Hemforth, Barbara (2001): Verstehenspräferenzen bei sprachlichen Mehrdeutigkeiten. In: Janetzko, Dietmar/Meyer, Herbert A./Hildebrandt, Michael (Hg.): Das experimentalpsychologische Praktikum im Labor und im WWW. Göttingen: Hogrefe.

MacDonald, Maryellen/Pearlmutter, Neal/Seidenberg, Marc (1994): The lexical nature of syntactic ambiguity resolutions. In: Psychological Review 101, S. 676-703.

Mitchell, Don C. (2004): On-line methods in language processing: Introduction and historical review. In: Carreiras, Manuel/Clifton Jr., Charles (2004) (ed.): The on-line study of sentence comprehension: Eyetracking, ERPs and beyond. New York: Psychology Press. S. 15-32.

Osterhout, Lee/Mobley, Linda A. (1995): Event-related brain potentials elicited by failure to agree. In: Journal of Memory and Language 34, S. 739-773.

Pickering, Martin J./Garrod, Simon (2004): Toward a mechanistic psychology of dialogue. In: Behavioral and Brain Sciences 27, S. 169-226. 
Rayner, Keith (Hg.) (1992): Eye movements and visual cognition: Scene perception and reading. New York: Springer.

Sanford, Anthony J./Garrod, Simon (1981): Understanding written language. Chichester: John Wiley.

Stevens, Stanley Smith (1957): On the psychophysical law. In: Psychological Review 64, S. $153-187$.

Tanenhaus, Michael K./Spivey-Knowlton, Michael/Eberhard, Kathleen/Sedivy, Julie C. (1995): Integration of visual and linguistic information in spoken-language comprehension. In: Science 268, S. 1632-1634.

Zwiserlood, Pienie/Boelte, Jens (2002): Worterkennung und -produktion. In Muesseler, Jochen/Prinz, Wolfgang (Hg.): Allgemeine Psychologie. Berlin: Spektrum. S. 546-587. 